JÚlio César Rosa e Silva ${ }^{1}$

Areana Diogo Nascimento ${ }^{2}$

ana Carolina Japur de Sá Rosa e Silva

Omero Benedicto Pol Neto ${ }^{3}$

Hermes de Freitas Barbosa ${ }^{1}$

Francisco JosÉ CANDIDO dOS ReIS

ANTÔNIO AlberTo NOGUEIRA ${ }^{5}$

Artigos originais

Palavras-chaves

Histeroscopia

Lidocaína/uso terapêutico Medição da dor

Keywords

Hysteroscopy

Lidocaine/therapeutic use Pain measurement

\title{
Uso do spray de lidocaína em histeroscopia diagnóstica
}

\author{
Use of lidocaine spray in diagnostic bysteroscopy
}

\section{Resumo}

OBJETIVO: determinar a eficácia do spray de lidocaína a 10\% aplicado no colo uterino antes da execução da histeroscopia diagnóstica para reduzir a dor e o desconforto causados pelo exame. MÉTODOS: Participaram do estudo 261 pacientes atendidas consecutivamente, entre março de 2004 e março de 2005. As pacientes foram randomizadas para dois grupos: um grupo recebendo aplicação tópica do spray de lidocaína (grupo lidocaína) e outro não recebendo nenhuma medicação antes do procedimento (grupo controle). Trinta miligramas de lidocaína spray a 10\% foram aplicados na superfície do colo uterino, sendo a histeroscopia iniciada cinco minutos depois. Imediatamente após o final do procedimento a paciente respondeu um questionário sobre dor e quantificou a intensidade da dor, em centímetros, usando uma escala analógica visual não graduada. Os testes $t$ não pareado, Mann-Whitney e $\chi^{2}$ foram aplicados na análise estatística, com nível de significância de 0,05. RESULTADOS: não houve diferença entre os grupos quanto à idade, paridade ou porcentagem de pacientes na menacme ou menopausa, ou quanto à indicação do procedimento e os achados histeroscópicos. Uma biópsia foi realizada em 57 das 132 pacientes no grupo lidocaína,

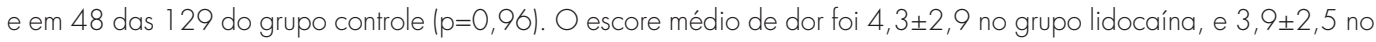
grupo controle $(p=0,2)$. Diferença no escore médio de dor foi observada somente na comparação entre pacientes na menacme e menopausa recebendo ou não a lidocaína spray, com p=0,01 e 0,04, respectivamente. CONCLUSÕES: o uso da lidocaína spray durante a histeroscopia diagnóstica não minimiza o desconforto e a dor das pacientes, e não deve ser utilizada.

\section{Abstract}

PURPOSE: to determine the efficacy of $10 \%$ lidocaine spray applied to the cervix before the procedure of diagnostic hysteroscopy, in order to reduce the painful process and the discomfort caused by the exam. METHODS: a total of 261 consecutive patients participated in the study, which was conducted from March 2004 to March 2005. The patients were randomly assigned to one of two groups: one group receiving topical lidocaine spray (lidocaine group - LdG) and the other, receiving no medication before the procedure (control group - CG). In the LdG patients, thirty milligrams of $10 \%$ lidocaine spray were applied to the surface of the cervix five minutes before hysteroscopy started. Immediately, after the end of the procedure, the patients from both groups were asked to respond to a questionnaire about pain and to quantify the pain, in centimeters, using a 10-cm non-graduated visual analog scale. The unpaired $t$ test, the MannWhitney test and the $\chi^{2}$ test were used for statistical analyses, considering p significant if lower than 0.05. RESULTS: there was no statistically significant difference between groups regarding age, parity or percentage of patients in menacme or menopause, or regarding the indications for the procedure and the hysteroscopic findings. A biopsy was necessary in 57 of the $132 \mathrm{LdG}$ patients and in 48 of the $129 \mathrm{CG}$ patients ( $\mathrm{p}=0.96)$. The mean pain score was $4.3 \pm 2.9 \mathrm{in} \mathrm{LdG}$ and $3.9 \pm 2.5$ in $C G(p=0.2)$. A difference in the mean pain score was observed only among patients in menacme and menopause receiving or not the lidocaine spray, with $p=0.01$ and $p=0.04$ respectively. CONCLUSIONS: the use of lidocaine spray during diagnostic hysteroscopy does not minimize the discomfort and pain of the patients and therefore should not be applied.

Correspondência:

Júlio César Rosa e Silva Departamento de Ginecologia e Obstetríicia Faculdade de Medicina de Ribeirão Preto Universidade de São Paulo - Avenida Bandeirantes, 3900 Campus da USP - CEP 14049-900 - Ribeirĩo Preto - SP Fone: (16) 3602-2589 E-mail: juliocrs@convex.com.br

Recebido

$14 / 11 / 2006$

Aceito com modificacōes 05/03/2007
Setor de Vídeo-endoscopia Ginecológica do Departamento de Ginecologia e Obstetrícia da Faculdade de Medicina da Universidade de São Paulo - USP - Ribeirão Preto (SP), Brasil.

' Médico Assistente do Departamento de Ginecologia e Obstetrícia da Faculdade de Medicina da Universidade de São Paulo - USP - Ribeirão Preto (SP), Brasil.

${ }^{2}$ Pós-graduanda do Departamento de Ginecologia e Obstetrícia da Faculdade de Medicina da Universidade de São Paulo - USP - Ribeirão Preto (SP), Brasil.

${ }^{3}$ Professor Doutor do Departamento de Cirurgia e Anatomia da Faculdade de Medicina da Universidade de São Paulo - USP - Ribeirão Preto (SP), Brasil.

4 Professor Associado do Departamento de Ginecologia e Obstetrícia da Faculdade de Medicina da Universidade de São Paulo - USP - Ribeirão Preto (SP), Brasil.

5 Professor Doutor do Departamento de Ginecologia e Obstetrícia da Faculdade de Medicina da Universidade de São Paulo - USP

- Ribeirão Preto (SP), Brasil. 


\section{Introdução}

A histeroscopia diagnóstica é hoje considerada como o melhor método de investigação da cavidade endometrial, sendo método de escolha na investigação de sangramento uterino anormal, anormalidades da cavidade endometrial visíveis pela ultra-sonografia ${ }^{1,2}$ e durante a propedêutica de infertilidade ${ }^{3}$. Apresenta vantagens em relação à dilatação do canal endocervical e curetagem, principalmente quanto ao custo, por não necessitar internação e uso de sala cirúrgica, e quanto à segurança, pois não necessita de anestesia geral, e tem sido mais eficaz na identificação de anormalidades da cavidade endometrial, principalmente anormalidades focais ${ }^{4,5}$.

Embora a histeroscopia diagnóstica possa ser realizada ambulatorialmente, muitas vezes é necessário o uso de anestesia local e não raras vezes a anestesia geral endovenosa é requisitada pela paciente pelo desconforto e dor ocasionados pelo exame, e cerca de $30 \%$ das pacientes submetidas a este procedimento sem qualquer tipo de anestesia referem ter dor severa ${ }^{6}$. Em geral, um bloqueio nervoso paracervical ou intracervical é realizado com objetivo de minimizar esse desconforto. Mesmo assim, cerca de $15 \%$ destas pacientes tem dor severa ${ }^{7}$, o que mostra uma falha destes métodos anestésicos, além dos riscos de sangramento, injeção intravascular e dor provocada pela administração do anestésico local ${ }^{8}$. Outra opção, de aplicação sistêmica, porém de baixo risco em termos de complicações, é o uso endovenoso de tramadol, que parece reduzir significativamente a dor durante o procedimento histeroscópico?

Algumas vias tópicas de administração de anestésicos, passíveis de serem realizadas ambulatorialmente, com finalidade de minimizar a dor e o desconforto causados pelo exame, já foram testadas com resultados diversos. A instilação transcervical de lidocaína $2 \%$ foi sugerida por alguns autores, mas alguns estudos mostraram que esta técnica de anestesia não apresenta bons resultados para prevenção da dor em procedimentos transcervicais como a histerossalpingografia ${ }^{10,11}$; entretanto, outros demonstraram ser este um método eficaz $^{12,13}$, embora doses diferentes tenham sido utilizadas. Outros anestésicos, como a benzocaína, também foram testados sem sucesso ${ }^{14}$.

O spray de lidocaína é uma alternativa muito usada para prevenir a dor durante procedimentos cirúrgicos menores na cavidade nasal e oral, e para realização de exames endoscópicos do aparelho digestivo e respiratório. Em ginecologia, pode ser utilizado em procedimentos cervicais e na mucosa genital e já foi testado em procedimentos histeroscópicos, mas com resultados contraditórios ${ }^{15}$.
O objetivo deste estudo foi verificar a eficácia de um spray de lidocaína $10 \%$ borrifado diretamente sobre o colo uterino antes da realização de histeroscopia diagnóstica para minimizar a dor e o desconforto causados pelo exame.

\section{Métodos}

Um total de 261 pacientes atendidas consecutivamente participaram do projeto desenvolvido no Hospital das Clínicas da Faculdade de Medicina de Ribeirão Preto da Universidade de São Paulo (USP), e foi aprovado pelo Comitê de Ética em Pesquisa desta Instituição. Todas as pacientes foram informadas do estudo, concordaram em participar e assinaram termo de consentimento pós-informado.

Foram, então, randomizadas aleatoriamente por um programa de computador em dois grupos, um que recebia lidocaína spray (Xylocaine; Astra Pharmaceuticals Ltd.) tópico (GLd) e outro que não recebia nenhuma medicação prévia ao procedimento (GC), sendo subdivididas em grupo lidocaína com pacientes na menacme (GLda) e na menopausa (GLdb), e grupo controle com pacientes na menacme (GCa) e na menopausa (GCb). Os critérios de inclusão foram que a paciente tivesse indicação de histeroscopia diagnóstica e que aceitasse participar do estudo, e os de exclusão foram hipersensibilidade a lidocaína e contra-indicações ao uso da medicação (epilepsia, doença hepática, uso de anti-depressivos tricíclicos e de inibidores da monoamino oxidase e alterações cardíacas e respiratórias graves), porém nenhuma paciente foi excluída após a randomização.

O estudo foi realizado no período de março de 2004 a março de 2005 com o seguinte procedimento padrão: a paciente era levada para a sala de exames, posicionada e realizadas três borrifadas de lidocaína spray $10 \%$ na superfície do cérvix uterino $(30 \mathrm{mg})$. Depois de cinco minutos o procedimento histeroscópico era iniciado. A histeroscopia diagnóstica foi realizada usando uma óptica de $4 \mathrm{~mm}$ com inclinação da lente de $30^{\circ}$ e canal de trabalho de $5 \mathrm{~mm}$ (Karl Storz, Germany). Foi utilizado o $\mathrm{CO}_{2}$ como meio de distensão da cavidade uterina, com fluxo de $50 \mathrm{~mL} / \mathrm{min}$ e pressão máxima de $200 \mathrm{mmHg}$. As biópsias endometriais foram realizadas sempre que uma lesão endometrial era identificada, fosse ela focal ou difusa, com auxílio de pinça histeroscópica de $2 \mathrm{~mm}$ (Karl Storz, Germany).

O sucesso do spray de lidocaína foi considerado quando a histeroscopia era realizada até o final e com boa visibilização da cavidade, sem a necessidade de outros procedimentos anestésicos. Imediatamente após o término do procedimento, a paciente respondia um 
breve questionário sobre dor e quantificava a dor sofrida durante o procedimento utilizando-se uma escala analógica visual de dor não graduada de $10 \mathrm{~cm}^{16}$. O questionário era aplicado por um pesquisador diferente daquele que realizava o exame e que não sabia a qual grupo de estudo pertencia a paciente. A intensidade da dor era medida em centímetros na escala analógica com auxílio de uma régua.

O cálculo estatístico foi realizado pelo programa GraphPad Prism ${ }^{\circledR} 2.0132$ Bit Executable (GraphPad Software Inc., San Diego, CA, EUA), utilizando-se os testes $t$ não pareado, Mann-Whitney e $\chi^{2}$, com nível de significância de 0,05.

\section{Resultados}

Participaram do estudo 261 pacientes divididas em dois grupos, sendo 132 no grupo que recebeu o spray de lidocaína (GLd) e 129 no grupo que não recebeu medicamentos antes da histeroscopia diagnóstica (GC). Não houve diferença estatística entre os grupos quando comparamos as variáveis idade, paridade e porcentagem de pacientes na menacme e na pós-menopausa (Tabela 1), nem quanto às indicações (Tabela 2) e achados histeroscópicos (Tabela 3). A biópsia foi necessária em 57 das 132 pacientes do GLd e em 48 das 129 pacientes do GC, não sendo observada diferença significante entre os grupos $(\mathrm{p}=0,96)$.

Em cinco pacientes do grupo que recebeu a lidocaína spray, foi necessária a aplicação de anestesia geral endovenosa por dor insuportável, sendo que no GC este número foi de quatro pacientes, não havendo diferença $(p=0,09)$. Podemos considerar, então, que houve sucesso na realização do exame em 127 de 132 pacientes do GLd $(96,2 \%)$ e em 125 de 129 do GC $(96,8 \%)$.

Quando analisamos o escore de dor das pacientes e comparamos entre os grupos não encontramos diferenças: no GLd o escore médio foi de 4,3 \pm 2 ,9, e no GC foi de $3,9 \pm 2,5(p=0,2)$, conforme Figura 1.

Se separamos os grupos em pacientes na menacme (GLda e GCa) e na pós-menopausa (GLdb e GCb), também não observamos diferença entre as usuárias de spray de lidocaína e as não usuárias. Pacientes na menacme que usaram lidocaína tiveram escore médio de dor de $4,8 \pm 3,0$ e as não usuárias de 4,2 22,7 , com $\mathrm{p}=0,15$. Já na menopausa estes números foram de 3,5 $\pm 2,6$ e $3,2 \pm 2,0$, respectivamente com $\mathrm{p}=0,9$.

Somente encontramos diferença significativa entre os escores médios de dor quando comparamos as pacientes na menacme com as pacientes na menopausa, usuárias ou não de spray de lidocaína: entre as usuárias de lidocaína, $\mathrm{p}=0,01$, e entre as não usuárias, $\mathrm{p}=0,04$.
Tabela 1 - Caracterização das pacientes submetidas à histeroscopia diagnóstica.

\begin{tabular}{lccc}
\hline $\begin{array}{l}\text { Características } \\
\text { das pacientes }\end{array}$ & $\begin{array}{c}\text { GLd } \\
(\mathbf{n}=132)\end{array}$ & $\begin{array}{c}\text { GC } \\
(\mathbf{n}=129)\end{array}$ & $\mathbf{p}$ \\
\hline Idade & $47,4 \pm 13,9$ & $45,8 \pm 13,8$ & NS \\
Nulípara & $18(13,6 \%)$ & $20(15,5 \%)$ & NS \\
Primípara & $32(24,2 \%)$ & $37(28,6 \%)$ & NS \\
Secundípara & $82(62,1 \%)$ & $72(55,8 \%)$ & NS \\
Menacme & $76(57,5 \%)$ & $82(63,5 \%)$ & NS \\
Menopausa & $56(42,5 \%)$ & $47(36,5 \%)$ & NS \\
\hline
\end{tabular}

GLd=grupo lidocaína; $G C=$ grupo controle; $N S=$ não significante.

Tabela 2 - Indicações para histeroscopia diagnóstica.

\begin{tabular}{lccc}
\hline Diagnósticos histeroscópicos & GLd (n=132) & GC ( $(n=129)$ & $\mathrm{p}$ \\
\hline Leiomioma & $7(5,4 \%)$ & $3(2,3 \%)$ & NS \\
Espessamento endometrial & $69(52,2 \%)$ & $58(45 \%)$ & NS \\
Infertilidade & $32(24,2 \%)$ & $29(22,5 \%)$ & NS \\
Controle pós-cirurgia & 0 & $3(2,3 \%)$ & NS \\
Pólipo endometrial & $19(14,4 \%)$ & $25(19,4 \%)$ & NS \\
Sangramento anormal & $3(2,3 \%)$ & $6(4,6 \%)$ & NS \\
Retirada de DIU & $2(1,5 \%)$ & $5(3,9 \%)$ & NS \\
\hline
\end{tabular}

GLd=grupo lidocaína; $G C=$ grupo controle; DIU=dispositivo intra-uterino; NS=não significante.

Tabela 3 - Distribuição dos achados histeroscópicos e número de biópsias endometriais realizadas.

\begin{tabular}{lccc}
\hline Diagnósticos histeroscópicos & GLd $(n=132)$ & GC ( $(n=129)$ & P \\
\hline Normal & $79(60 \%)$ & $75(58,1 \%)$ & NS \\
Pólipo endometrial & $32(24,2 \%)$ & $32(24,8 \%)$ & NS \\
Leiomioma & $12(9,1 \%)$ & $8(6,3 \%)$ & NS \\
Sinéquia ułerina & $4(3 \%)$ & $3(2,3 \%)$ & NS \\
Câncer & $3(2,2 \%)$ & $6(4,6 \%)$ & NS \\
DIU intra-ułerino & $2(1,5 \%)$ & $5(3,9 \%)$ & NS \\
Biópsia endometrial & $57(43,2 \%)$ & $48(37,2 \%)$ & NS \\
\hline
\end{tabular}

$\mathrm{GLd}=$ grupo lidocaína; $\mathrm{GC}=$ grupo controle; $\mathrm{DIU}=$ dispositivo intra-uterino; NS=não significante.

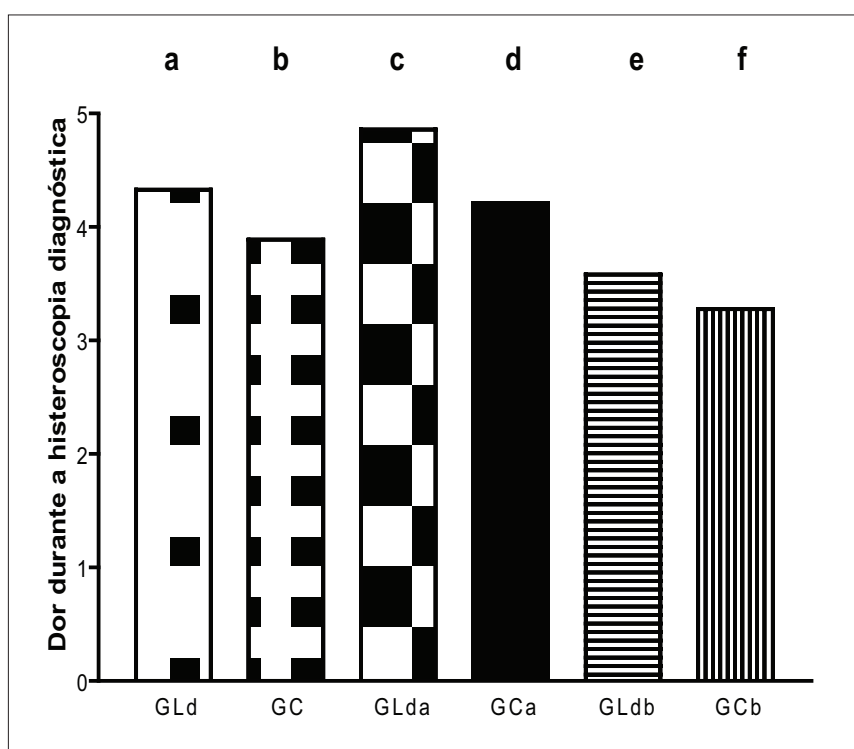

GLd=grupo lidocaína; GC=grupo controle; GLda=grupo lidocaína no menacme; $\mathrm{GCa}$ =grupo controle no menacme; $\mathrm{GLdb}=$ grupo lidocaína na pós-menopausa; GCb=grupo controle na pós-menopausa. $a=b ; c=d ; e=f ; c>e(p=0,01) ; d>f(p=0,04)$.

Figura 1 - Média dos escores de dor referida pelas pacientes nos diferentes grupos. 
Nenhum efeito adverso ou desconforto provocado pela aplicação da lidocaína foi relatado.

\section{Discussão}

Nosso estudo não mostrou eficácia do uso do spray de lidocaína para diminuição da dor e do desconforto da paciente durante a histeroscopia diagnóstica, confirmando dados de estudos anteriores com injeção intracervical $^{17}$, paracervical $^{8}$ e mesmo anestesia tópica ${ }^{18,19}$, que também não mostraram esse benefício. No entanto, outros estudos encontraram melhora significativa na diminuição da dor com uso de lidocaína spray ${ }^{20,21}$ e com a instilação transcervical (intracavitária) de lidocaína líquida $2 \%{ }^{12,13}$.

Resultados semelhantes aos encontrados neste estudo já foram obtidos. No entanto, houve diferença significativa quanto à dor somente durante a colocação da pinça Pozzi na cérvix uterina, não se encontrando diferença para a colocação da óptica no canal endocervical, durante a histeroscopia, biópsia endometrial ou cinco minutos após o término do procedimento ${ }^{22}$.

A histeroscopia diagnóstica é um procedimento suportado pela maioria das pacientes, como já demonstrado anteriormente ${ }^{7}$, mesmo quando é realizada a biópsia endometrial. Sem o emprego de nenhum procedimento anestésico adicional, nossa porcentagem de sucesso ao realizar este procedimento com e sem uso de lidocaína spray foi muito alto, 96,2 e 96,8\%, respectivamente, confirmando dados publicados anteriormente ${ }^{7,20}$. Eventualmente, o uso de drogas analgésicas, como o tramadol endovenoso, pode ser feito com sucesso principalmente em casos em que a queixa de dor for mais acentuada?

A administração da lidocaína spray tem muitas vantagens em relação à injeção intracervical ou paracervical, porque não está associada a dor para aplicação, riscos de sangramento, de injeção intravascular de lidocaína ou de infecção, porém não vemos utilidade no seu uso porque não observamos melhora significativa na diminuição da dor durante o procedimento. Talvez isso decorra do fato que os índices de dor durante o procedimento foram baixos (4,3 e 3,9 para os GLd e GC, respectivamente), o que é considerado como dor leve. Mesmo quando separamos as pacientes em dois grupos, menacme e pós-menopausa, não encontramos diferença entre os grupos que usaram e os que não usaram lidocaína spray, mostrando que isto não deve influenciar no resultado.

Porém, observamos em nossos resultados que as pacientes na menacme referem dor mais intensa que as pacientes menopausadas. Isso pode ocorrer por questões de temperamento e ansiedade das pacientes na menacme, que na sua maioria tinham indicação deste exame por infertilidade ou por sangramento uterino anormal, enquanto que as pacientes na menopausa na sua maioria eram assintomáticas e tiveram sua indicação associada a achados ultra-sonográficos ${ }^{22}$.

Com estes dados, concluímos que o uso da lidocaína spray durante o procedimento de histeroscopia diagnóstica não minimiza o desconforto e a dor que a paciente sente e, portanto, não traz benefícios quando de sua utilização.
1. Goldrath MH, Sherman Al. Office hysteroscopy and suction curettage: can we eliminate the hospital diagnostic dilatation and curettage? Am J Obstet Gynecol. 1985;152(2): 220-9.

2. Nagele F, O'Connor H, Davies A, Badawy A, Mohamed H, Magos A. 2500 Outpatient diagnostic hysteroscopies. Obstet Gynecol. 1996;88(1):87-92.

3. de Sa Rosa e Silva AC, Rosa e Silva JC, dos Reis FJ, Nogueira AA, Ferriani RA. Routine office hysteroscopy in the investigation of infertile couples before assisted reproduction. J Reprod Med. 2005;50(7):501-6.

4. Gimpelson RJ, Rappold HO. A comparative study between panoramic hysteroscopy with directed biopsies and dilatation and curettage. Am J Obstet Gynecol. 1988; 158(3 Pt 1):489-92.

5. Loffer FD. Hysteroscopy with selective endometrial sampling compared with D\&C for abnormal uterine bleeding: the value of a negative hysteroscopic view. Obstet Gynecol. 1989;73(1):16-20.
6. De laco P, Marabini A, Stefanetti M, Del Vecchio C, Bovicel L. Acceptability and pain of outpatient hysteroscopy. J Am Assoc Gynecol Laparosc. 2000;7(1):71-5.

7. De Jong P, Doel F, Falconer A. Outpatient diagnostic hysteroscopy. Br J Obstet Gynaecol. 1990;97(4):299-303.

8. Vercellini P, Colombo A, Mauro F, Oldani S, Bramante T, Crosignani PG. Paracervical anesthesia for outpatient hysteroscopy. Fertil Steril. 1994;62(5): 1083-5.

9. Floris S, Piras B, Orru M, Silvetti E, Tusconi A, Melis F, et al. Efficacy of intravenous tramadol treatment for reducing pain during office diagnostic hysteroscopy. Fertil Steril. 2007;87(1):147-51.

10. Costello MF, Horrowitz S, Steigrad S, Saif N, Bennett $M$, Ekangaki A. Transcervical intrauterine topical local anesthetic at hysterosalpingography: a prospective, randomized, double-blind, placebo-controlled trial. Fertil Steril. 2002;78(5): 111 16-22.

11. Frishman GN, Spencer PK, Weitzen S, Plosker S, Shafi F. The use of intrauterine lidocaine to minimize pain during hysterosalpingography: a randomized trial. Obstet Gynecol. 2004;103(6):1261-6. 
12. Hui SK, Lee L, Ong C, Yu V, Ho LC. Intrauterine lignocaine as an anaesthetic during endometrial sampling: a randomised double-blind controlled trial. Br J Obstet Gynaecol. 2006; $113(1): 53-7$.

13. Guney M, Oral B, Mungan T. Efficacy of intrauterine lidocaine for removal of a "lost" intrauterine device: a randomized, controlled trial. Obstet Gynecol. 2006;108(1):119-23.

14. Einarsson Jl, Henao G, Young AE. Topical analgesia for endometrial biopsy: a randomized controlled trial. Obstet Gynecol. 2005; 106(1):128-30

15. Van der Burght M, Schonemann NK, Laursen JK, Arendt-Nielson L, Bierring P. Onset and duration of hypoalgesia following application of lidocaine spray on the genital mucosa. Acta Obstet Gynecol Scand. 1994;73(10):809-11.

16. Woodforde JM, Merskey H. Some relationships between subjective measures of pain. J Psychosom Res. 1972;16(3):173-8.

17. Broadbent JAM, Hill NC, Molnar BG, Rolfe KJ, Magos AL. Randomized placebo controlled trial to assess the role of intracervical lignocaine in outpatient hysteroscopy. Br J Obstet Gynaecol. 1992;99(9):777-9.

18. Hasson HM. Topical uterine anesthesia: a preliminary report. Int J Gynaecol Obstet. 1977;15(3):238-40.

19. Cicinelli E, Didonna T, Ambrosi G, Schonauer LM, Fiore G, Matteo MG. Topical anaesthesia for diagnostic hysteroscopy and endometrial biopsy in postmenopausal women: a randomised placebo-controlled double-blind study. Br J Obstet Gynaecol. 1997; 104(3):316-9.

20. Soriano D, Ajaj S, Chuong T, Deval B, Fauconnier A, Darai E. Lidocaine spray and outpatient hysteroscopy: randomized placebocontrolled trial. Obstet Gynecol. 2000;96(5 Pt 1):661-4.

21. Wieser F, Kurz C, Wenzl R, Albrecht A, Huber JC, Nagele F. Atraumatic cervical passage at outpatient hysteroscopy. Fertil Steril. 1998;69(3):549-51

22. Davies A, Richardson RE, O'Connor H, Baskett TF, Nagele F, Magos AL. Ligdocaine aerosol spray in outpatient hysteroscopy: a randomized double-blind placebo-controlled trial. Fertil Steril. 1997;67(6):1019-23. 